\title{
NANO-ENGINEERING OF ACTIVE METAMATERIALS
}

\section{Larry Dalton}

UNIVERSITY OF WASHINGTON

$10 / 29 / 2014$

Final Report 


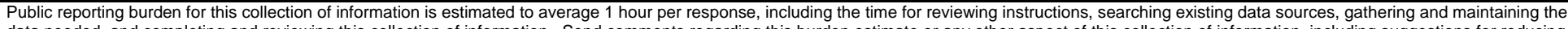

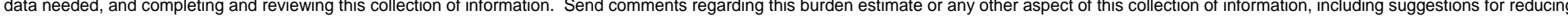

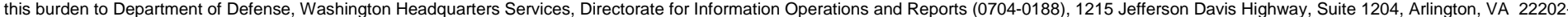

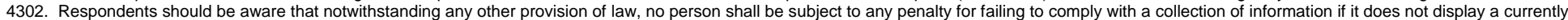
valid OMB control number. PLEASE DO NOT RETURN YOUR FORM TO THE ABOVE ADDRESS.

\begin{tabular}{l|l|l} 
1. REPORT DATE (DD-MM-YYYY) & 2. REPORT TYPE & 3. DATES COVERED (FrOm - To)
\end{tabular}

\begin{tabular}{l|l}
$24 / 10 / 2014$ & Final Report \\
\hline
\end{tabular}

\section{TITLE AND SUBTITLE}

Nano-Engineering of Active Metamaterials

From 15/09/2009 to $14 / 09 / 2014$

5a. CONTRACT NUMBER

FA9550-09-1-0682

5b. GRANT NUMBER

5c. PROGRAM ELEMENT NUMBER

\section{AUTHOR(S)}

Larry R. Dalton

Bruce H. Robinson 5d. PROJECT NUMBER

5e. TASK NUMBER

5f. WORK UNIT NUMBER

8. PERFORMING ORGANIZATION REPORT NUMBER

$61-8594$
Universityof Washington

Office of Sponsored Programs

4333 Brooklyn Avenue NE

Seattle, WA 98105-1016

\section{SPONSORING / MONITORING AGENCY NAME(S) AND ADDRESS(ES)}

Dr. Charles Y.-C. Lee

875 N. Randolph Steet, Suite 325

Room 3112

Air Force Office of Scientific Research

Arlinaton. VA 22203-1954

12. DISTRIBUTION I AVAILABILITY STATEMENT

Distribution A - Approved for Public Release
10. SPONSOR/MONITOR'S ACRONYM(S)

AFOSR

11. SPONSOR/MONITOR'S REPORT NUMBER(S)

\section{SUPPLEMENTARY NOTES}

\section{ABSTRACT}

Nonlinear optical and gain materials are incorporated into both metallic/semiconductor and dielectric (e.g., organic) metamaterial architectures for active control of light. Potential applications include imaging, sensing, and information management (e.g., telecommunications). New material structures are based on nonlinear optical and gain materials integrated with silicon photonic and plasmonic materials, which are capable of generating substantial local field enhancement and upon one-, two-, and three dimensional metamaterials. Nonlinear optical and gain materials integrated metamaterial architectures include laser dyes and amplifier materials and both second and third order nonlinear optical materials. Both organic and inorganic active materials are explored to achieve desired wavelength control of phenomenon and realize the desired optical effects. Mechanisms for efficient coupling of light into and out of metamaterials (another recognized problem) are investigated.

\section{SUBJECT TERMS}

Active metamaterials, nonlinear optics, organic nonlinear optical materials, silicon photonics, plasmonics, theory-guided design

\begin{tabular}{|c|c|c|c|c|c|}
\hline \multicolumn{3}{|c|}{ 16. SECURITY CLASSIFICATION OF: } & $\begin{array}{l}\text { 17. LIMITATION } \\
\text { OF ABSTRACT }\end{array}$ & $\begin{array}{l}\text { 18. NUMBER } \\
\text { OF PAGES }\end{array}$ & $\begin{array}{l}\text { 19a. NAME OF RESPONSIBLE PERSON } \\
\text { Larry R. Dalton }\end{array}$ \\
\hline $\begin{array}{l}\text { a. REPORT } \\
\text { Unclassified }\end{array}$ & $\begin{array}{l}\text { b. ABSTRACT } \\
\text { Unclassified }\end{array}$ & $\begin{array}{l}\text { c. THIS PAGE } \\
\text { Unclassified }\end{array}$ & Unclassified & 17 & $\begin{array}{l}\text { 19b. TELEPHONE NUMBER (include area } \\
\text { code) } \\
\text { (360) } 981-4575\end{array}$ \\
\hline
\end{tabular}


FINAL TECHNICAL REPORT (AFOSR-FA9550-09-1-0682)-Nano-Engineering of Active Metamaterials: Publications related to this AFOSR contract (2009-2014) are listed as references [1-84].

Executive Summary: Metamaterial phenomena can be considered to be an example of "hybrid material optics" (based on the integration of metals, semiconductors, and organic macromolecular materials). Such integration has been used to realize and exploit sub-wavelength (sub- $\lambda$ ) photonic circuitry. Concentrating light and radiofrequency fields in such nanometer-scale device architectures permits dramatic enhancement of nonlinear optical phenomena relevant to transformative improvements in telecommunication, computing, and sensing. There are two critical elements to advancing metamaterial/hybrid material optics performance: (1) Theory-guided design of new optical and nonlinear optical materials and (2) development of new device architectures and resolution of the challenges presented by those new architectures. In the coarse of this research, the performance of organic electrooptic material has been improved to approximately $500 \mathrm{pm} / \mathrm{V}$ in thin film architectures and 200-300 pm/ V in sub- $\lambda$ devices. The improvements in materials have been combined with new device architectures to realize record drive voltage-length factors (50 V-micron), digital and analog bandwidths (>100 Gbit/s; > $100 \mathrm{GHz}$ ), power efficiencies ( $<1$ femtojoule/bit), and device footprints $\left(<5\right.$ micron $\left.^{2}\right)$. New materials and device architectures are being transitioned to record setting demonstrations in telecommunications and sensing.

Multi-Scale Theoretical Developments and Applications: There are two aspects to the multi-scale (quantum/statistical mechanics) theoretical efforts of this research: (1) Development and application of time-dependent density functional theory (TD-DFT) and Moller-Plesset (MPn) perturbation theory, which permit explicit treatment of applied and molecular (dielectric permittivity) time-dependent fields and (2) coarse-grained Monte Carlo/molecular dynamics computational methods capable of simulating order (e.g., lattice dimensionality and acentric order) and dynamics (e.g., viscoelasticity) in macromolecular materials containing electro-optic chromophores. These two approaches combine to form a multi-scale computational method capable of providing critical guidance to the development of new organic electroactive (including electro-optic) materials.

Multi-dimensional methods have permitted quantitative simulation of linear and nonlinear optical properties and dielectric permittivity including those of dipolar liquids, dendrimers, polymers, and inorganic materials. Theory is bridging the conceptual gap between various methods of preparing organic electro-optic materials (electric field poling, crystal growth, sequential synthesis).

Development and Application of New Characterization Tools: Critical to the development of reliable theoretical guidance and definition of critical structure/function relationships is the ability to accurately measure linear and nonlinear optical properties, thermal and photochemical stability, etc. We have assembled a unique tool set providing the capability for measurement of molecular first hyperpolarizability by wavelength-agile, femtosecond HRS and by EFISH [11,50], electro-optic activity by modified Teng-Man Ellipsometry and attenuated total reflection (ATR) [11,50], acentric order parameters by variable angle polarized absorption spectroscopy (VAPAS) [11,26] and variable angle spectroscopic ellipsometry (VASE) [11] and by atomic resolution techniques including two photon fluorescence microscopy [1,21], and measurement of photochemical stability at telecommunication wavelengths by pump-probe methods [4,11].

Development and Application of New Synthesis and Processing Methodologies: We have pioneered microwave-assisted synthesis as applied to the production of organic electroactive materials and have been a leader in developing new lattice hardening chemistries, particularly Diels-Alder/Retro-Diels-Alder cycloaddition reactions, which have yielded organic electro-optic materials with glass transition temperatures exceeding $200^{\circ} \mathrm{C}[11,34,38,47]$. Our laboratory has pioneered the fabrication of complex device structures, such as coupled multi-ring microresonators by soft and nanoimprint lithography and we 
have been actively involved in the fabrication of conformal and flexible devices by lift-off techniques. Recently, we have introduced laser-assisted electric field poling [11,55] as a means of improving electrooptic activity while maintaining low optical loss and even more recently we have demonstrated that nanoscopic metal oxide surface coatings and surface functionalization with an oriented chromophore monolayer can lead to improved optical nonlinearity. We have also pioneered the incorporation of organic nonlinear optical materials into silicon photonic waveguide and nano-slot waveguide device structures. This integration has included utilization of doped silicon as poling and drive electrodes. The integration of organic NLO materials with silicon photonic structures has been extended to the demonstration of Reconfigurable Optical Add/Drop Multiplexer (ROADM) devices (for chipscale information routing) carried out in collaboration with Boeing. A significant accomplishment of our theoretical simulations of device performance is the prediction of concentration of light in nanoscopic slots etched into silicon photonic waveguides. These slots permit nearly lossless transition of light from high index of refraction silicon into lower index of refraction organic nonlinear optical materials for active control of light.

Photostability Studies: In a collaborative study with Professor Peter Gunter of the ETH, Zurich, we have analyzed the photochemical kinetics (employing optical pump powers in the range $400 \mathrm{~mW}-1 \mathrm{~W}$ for accelerated testing) of dozens of organic electro-optic materials. Improved analysis and measurement protocols have been developed. Singlet oxygen chemistry is the found to define the dominant photodegradation mechanisms and packaging of hardened materials has yielded photostability figure-ofmerit values $(\mathrm{B} / \sigma$ or the number of absorbed photons required to produce a damage event) as large as $5000 \times 10^{32} \mathrm{~m}^{-2}$ (which would yield more than 100 years of operation for normal telecommunication power levels). Our studies have been particularly useful in demonstrating the dependence of photostability on chromophore structure. Here we have space to illustrate only one example (Fig. 8) but photostability can be typically varied by up to two orders of magnitude with simple variation of chromophore structure. Note that chromophores in dendrimers are usually more stable than the same chromophore in polymer composites consistent with reduced oxygen accessability in the dendrimer material lattice. Lattice hardening (crosslinking) and use of singlet oxygen quenchers lead to further multiple order of magnitude improvements of photostability. As with researchers at Corning , we observe up to 4-5 orders of magnitude variation in photostability for the same parent chromophore $\pi$-electron structure as a function of derivativation of that core structure, lattice hardening, use of quenchers, and packaging to exclude oxygen. While photostability issues are not as serious for organic electro-optic materials as for organic light emitting device and photovoltaic materials (because the operational light is off resonance and current flow is minimum in EO applications), packaging will still likely be required to achieve 10 year operational lifetimes, particularly if optical power levels exceed typical telecommunication powers.

In summary, we have established the world's leading facility for the characterization and analysis of the photostability of organic electro-optic materials, in both thin films and devices, for a wide range of operational optical wavelengths including both telecommunication bands. We have carried out definition of mechanisms of photodegradation for a wide range of materials including chromophore/polymer composites; chromophore-containing dendrimers, polymers, and dendronized polymers; and for binary chromophore organic glasses. We have also investigated a wide range of processing conditions including examining the effects of lattice hardening, the addition of singlet oxygen quenchers, material packaging, etc. We have all the necessary facilities and expertise to carry out photostability measurements and improvements to meet metrics proposed in the following sections of this proposal.

Implementation and Demonstration of Prototype Devices: The two most commonly implemented prototype device structures are shown in the accompanying figures. Mach Zehnder (MZ), In-PhaseQuadrature (IQ), and Phase Modulators are the most commonly employed modulator configurations utilized for information management in telecommunications, computing, and sensing applications. 
Incorporating OEO materials into these device structures we have achieved record performance for information management. The results can be summarized as follows for silicon-organic hybrid (SOH) and plasmonic silicon-organic hybrid (PSOH) devices:

For SOH devices, the following performance has been achieved for devices with analog bandwidths greater than $100 \mathrm{GHz}$ and digital bandwidths greater than $100 \mathrm{Gbit} / \mathrm{s}$.

Voltage-Length Parameter: 0.5 V-mm; Digital Power Consumption: < 1 fJ/bit; Insertion Loss: < $10 \mathrm{~dB}$; Device Footprint: $<0.25 \mathrm{~mm}^{2}$

For PSOH devices, the following performance has been achieved for devices with analog bandwidths greater than $100 \mathrm{GHz}$ and digital bandwidths greater than $100 \mathrm{Gbit} / \mathrm{s}$

Voltage-Length Parameter: 50 V-micron; Digital Power Consumption: < 13 fJ/bit; Insertion Loss: < 12 dB; Device Footprint: $<25$ micron $^{2}$

\section{REFERENCES (AFOSR-FA9550-06-1-0682 relevant publications)}

1. Haishan Sun, Antao Chen, and Larry R. Dalton, "Multiple Slot Waveguides for Enhanced Biochemical Sensing," IEEE/LEOS International Conf. on Optical MEMS and Nanophotonics,” pp. 21-22, DOI:

10.1109/OMEMS.2009.5338626 (2009).

2. $\quad$ Larry R. Dalton, “Electro-optic polymers,” McGraw-Hill Yearbook of Science \& Technology 2009, McGraw-Hill, New York, 2009, pp. 99-102.

3. Haishan Sun, Antao Chen, and Larry R. Dalton, “A Reflective Microring Notch Filter and Sensor,” Optics Express, 17 (13), 10731-10737 (2009); also published on-line in The Virtual Journal for Biomedical Optics, Vol. 4, Issue 8, July 30, 2009, (http://www.opticsinfobase.org/vjbo/virtual_issue.cfm).

4. Haishan Sun, Antao Chen, and Larry R. Dalton, "Enhanced Evanescent Confinement in Multiple-Slot Waveguides and Its Application in Biochemical Sensing,” IEEE Photonics Journal, 1(1), 48-57, DOI: 10.1109/JPOT.2009.2025602 (2009).

5. $\quad$ Stephanie J. Benight, Denise H. Bale, Benjamin C. Olbricht, and Larry R. Dalton, "Organic Electro-Optics: Understanding Material Structure/Function Relationships and Device Fabrication Issues,” J. Mater. Chem., 19, 74667475, DOI: 10.1039/b905368 (2009).

6. Larry R. Dalton, “Theory-Inspired Development of Organic Electro-Optic Materials,” Thin Solid Films, 518, 428-431, DOI: 10.1016/j.tsf.2009.07.00 (2009).

7. Larry R. Dalton, Philip Sullivan, Denise H. Bale, Scott Hammond, Benjamin C. Olbricht, Harrison Rommel, Bruce E. Eichinger, and Bruce H. Robinson, "Organic Photonic Materials," in Tutorials in Complex Photonic Media, M. Noginov, M. W. McCall, G. Dewar, and N. I. Zheludev, eds., SPIE Press, Bellingham, WA (2009) Chapter 16, pp 535-574.

8. Haishan Sun, Antao Chen, Attila Szep, and Larry R. Dalton, "Efficient Fiber Coupler for Vertical Silicon Slot Waveguides,” Optics Express, 17, 22571-22577 (2009).

9. P. A. Sullivan, H. L. Rommel, Y. Takimoto, S. R. Hammond, D. H. Bale, B. C. Olbricht, Y. Liao, J. Rehr, B. E. Eichinger, A. K.-Y. Jen, P. J. Reid, L. R. Dalton, and B. H. Robinson, "Modeling the Optical Behavior of Complex Organic Media: From Molecules to Materials,” J. Phys. Chem. B, 113 (47), 15581-15588, DOI: 10.1021/jp908057 (2009).

10. Larry R. Dalton, David Lao, Benjamin C. Olbricht, Stephanie Benight, Denise H. Bale, Joshua A. Davies, Todd Ewy, Scott R. Hammond, and Philip A. Sullivan, "Theory-inspired development of new nonlinear optical materials and their integration into silicon photonic circuits and devices,” Opt. Mater., 32, 658-668 DOI:10.1016/j.optmat.2009.02.002 (2009).

11. Larry R. Dalton, Philip A. Sullivan, and Denise H. Bale, "Electric Field Poled Organic Electro-Optic Materials: State of the Art and Future Prospects," Chemical Reviews, 110, 25-55, DOI: 10.1021/cr9000428 (2010). (Citations = 72).

12. Philip A. Sullivan and Larry R. Dalton, “Theory-Inspired Development of Organic Electro-Optic Materials,” Accounts of Chemical Research, 43 (1), 10-18, DOI: 10.1021/ar800264w (2010).

13. Larry R. Dalton, "Electro-Optical Applications," in Encyclopedia of Polymer Science and Technology, (H. F. Mark, ed.) John Wiley \& Sons, New York, January 29, 2010, DOI: 10.1002/0471440264.pst110.pub2.

14. Ran Ding, T. Baehr-Jones, L. Yang, R. Bojko, J. Witzens, H. Su, J. Luo, S. Benight, P. Sullivan, J. Fedeli, M. Fournier, L. R. Dalton, A. Jen, and M. Hochberg, “A Low V V Moudlator With GHz Bandwidth Based on an Electro- 
Optic Polymer-Clad Silicon Slot Waveguide,” Proc. IEEE $7^{\text {th }}$ International Conf. on Group IV Photonics (GFP), pp. 201-203, DOI: 10.1109/GROUP4.2010.5643391 (2010).

15. L. R. Dalton, P. A. Sullivan, D. Bale, B. Olbricht, J. Davies, S. Benight, I. Kosilkin, B. H. Robinson, B. E. Eichinger, and A. K. Y. Jen, "Organic Electro-Optic Materials: Understanding Structure/Function Relationships Critical to the Optimization of Electro-Optic Activity,” in Organic Thin Films for Photonics Applications, eds. W. Herman and S. Foulger (ACS Symposium Series, Washington DC, 2010) Chapter 2, pp. 13-33, DOI: 10.1021/bk-2010-1039.ch002.

16. X. H. Zhou, J. Luo, T. D. Kim, S. H. Jang, R. M. Overney, L. R. Dalton, and A. K. Y. Jen, "Molecular Design and Supramolecular Organization of Highly Efficient Nonlinear Optical Chromophores for Exceptional Electro-Optic Properties," in Organic Thin Films for Photonics Applications, eds. W. Herman and S. Foulger (ACS Symposium Series, Washington DC, 2010) Chapter 4, pp. 51-66, DOI: 10.1021/bk-2010-1039.ch004.

17. Anna Pyayt, Antao Chen, and Larry R. Dalton, "Field-Induced Guiding Optical Devices Made from Electro-Optic Polymers," Applied Optics, 49, 892-896 DOI:10.1364/AO.49.000892 (2010).

18. Ran Ding, Tom Baehr-Jones, Yang Liu, Rick Bojko, Jeremy Witzens, Su Huang, Jingdong Luo, Stephanie Benight, Phillip Sullivan, Jean-Marc Fedeli, Maryse Fournier, Larry Dalton, Alex Jen, and Michael Hochberg, "Demonstration of a Low $\mathrm{V}_{\pi} \mathrm{L}$ Modulator with GHz Bandwidth Based on an Electro-optic Polymer-Clad Silicon Slot Waveguide,” Optics Express, 18 (15), 15618-15623 DOI:10.1364/OE.18.015618 (2010).

19. J. G. Grote, L. R. Dalton, P. Sullivan, B. H. Robinson, B. Eichinger, A. K.-Y. Jen, S. Benight, I. Kosilkin, and D. H. Bale, "Definition of Critical Structure/Function Relationships and Integration Issues for Organic Electro-Optic Materials," Nonlinear Optics and Quantum Optics, 40, 15-26 (2010).

20. Stephanie J. Benight, Lewis E. Johnson, Robin Barnes, Benjamin C. Olbricht, Denise H. Bale, Bruce E. Eichinger, Larry R. Dalton, Philip A. Sullivan, and Bruce H. Robinson, "Reduced Dimensionality in Organic Electro-Optic Materials,” J. Phys. Chem. B, 114(37), 11949-11956, DOI: 10.1021/jp1022423 (September 17, 2010 issue cover article).

21. Seong-Ku Kim, Nathan Sylvain, Stephanie J. Benight, Ilya Kosilkin, Denise H. Bale, Bruce H. Robinson, Kevin Geary, Alex K.-Y. Jen, William, H. Steier, Harold R. Fetterman, Pierre Berini, and Larry R. Dalton, “Active Plasmonic and Metamaterials and Devices," Proc SPIE, 7754, 775403 1-10, DOI: 10.1117/12.862061 (2010).

22. Stephanie J. Benight, Lewis E. Johnson, Daniel B. Knorr, Arumugasamy Elangovan, Ilya Kosilkin, Benjamin C. Olbricht, Philip A. Sullivan, Denise H. Bale, Bruce H. Robinson, Rene M. Overney, Alex K.-Y. Jen, William H. Steier, Seong-Ku Kim, Harold R. Fetterman, Dennis W. Prather, and Larry R. Dalton, "Theory-Guided Enhancement of Poling Efficiency of Organic Electro-Optic Materials,” Proc. SPIE, 7774, 77740F 1-12, DOI: 10.1117/12.862059 (2010).

23. Seong-ku Kim, Harold R. Fetterman, K. Geary, Pierre Berini, and Larry R. Dalton, “Au and Ag Nano-Particle Embedded Plasmonic Metal-Slotted Polymer Electro-Optic Waveguide Modulator,” Proc. 2011 Conf. on Laser and Electro-Optics, pp. 1-3 (2011).

24. H. Figi, D. H. Bale, A. Szep, L. R. Dalton, and A. Chen, "Horizontally Slotted Electro-Optic Waveguides Featuring an Organic Crystalline Slot Material,” Proc. Conf. on Lasers and Electro-Optics Europe and 23th European Quantum Electronics Conf., DOI: 20.1109/CLEOE.2011.5942706 (2011).

25. Seong-ku Kim, Wei Liu, Harold R. Fetterman, Qibing Pei, and Larry R. Dalton, "Suppression of Intermodulation Distortion (IMD) in Coherent Systems Using Polymeric Dual Parallel Mach-Zehnder Modulators,” Proc. Conf. on Lasers and Electro-Optics Europe and 23th European Quantum Electronics Conf., DOI:

20.1109/CLEOE.2011.5942706 (2011).

26. Benjamin C. Olbricht, Philip A. Sullivan, Joshua A. Davies, Peter C. Dennis, Jeffrey T. Hurst, Lewis E. Johnson, Denise H. Bale, Stephanie J. Benight, James N. Hilfiker, Antao Chen, Bruce E. Eichinger, Philip J. Reid, Larry R. Dalton, and Bruce H. Robinson, "Measuring Order in Contact-Poled Organic Electro-Optic Materials with Variable Angle Polarization-Referenced Absorption Spectroscopy (VAPRAS),” J. Phys. Chem. B, 115(2), 231-241, DOI: 10.1021/jp107995t (2011).

27. Larry R. Dalton, Stephanie J. Benight, Lewis E. Johnson, Daniel B. Knorr Jr., Ilya Kosilkin, Bruce E. Eichinger, Bruce H. Robinson, Alex Jen, and Rene Overney, "Systematic Nano-Engineering of Soft Matter Organic ElectroOptic Materials,” Chem. Mater., 23(3), 430-445, DOI: 10.1021/cm102166 (2011).

28. Larry R. Dalton, Alex K.-Y. Jen, Bruce H. Robinson, Rene Overney, Stephanie J. Benight, Daniel B. Knorr, Jr., Seongku Kim, Harold Fetterman, and Cheng Zhang, "Optimization of Organic NLO Materials for Integration with Silicon Photonic, Plasmonic (Metal Optics), and Metamaterial Devices,” Proc. SPIE, 7935, 793502-1-15, DOI: org/10.1117/12.881264 (2011).

29. Stefan Meister, Dawid Schweda, Marcus Dziedzina, Ronny Juhre, Aws Al-Saadi, Bulenet A. Franke, Bernd Grimm, Sigurd K. Chrader, Stephanie J. Benight, Denise H. Bale, Ilya Kosilkin, Larry R. Dalton and Hans J. Eichler, "Filters and electro-optic modulators on fiber end-faces," Proc. SPIE, 7913, 79130L-1-8 (2011).

30. Denise Bale, Wenkel Liang, Larry Dalton, Philip Reid, Bruce Robinson, Bruce Eichinger, and Xiasong Li, "Dielectric Dependence of the First Molecular Hyperpolarizability for Electro-Optic Chromophores,” J. Phys. Chem. B, 115(13), 3505-3513, DOI: 10.1021/jp109870y (2011).

31. Weiwei Sun, Zhaohong Wang, Antao Chen, Ilya Kosilkin, Denise Bale, and Larry R. Dalton, "Electro-Optic thin 
Films of Organic Nonlinear Optic Molecules Aligned Through Vacuum Deposition,” Optics Express, 19(12), 1118911195, DOI: 10.1364/OE.19.011189 (2011).

32. Zhaohong Wang, Weiwei Sun, Antao Chen, Ilya Kosilkin, Denise Bale, and Larry R. Dalton, "Organic Electro-Optic Thin Films by Simultaneous Vacuum Deposition and Laser-Assisted Poling,” Optics Lett., 36(15), 2853-2855, DOI: 10.1364/OL.36.002853 (2011).

33. Harry Figi, Denise H. Bale, Attila Szep, Larry R. Dalton, and Antao Chen, "Electro-Optic Modulation in Horizontally Slotted Silicon/Organic Crystal Hybrid Devices,” J. Opt. Soc. Amer. B, 28(9), 2291-2300, DOI: 10.1364/JOSAB.28.002291 (2011).

34. Seongku Kim, Qibing Pei, H. R. Fetterman, B. C. Olbricht, and L. R. Dalton, "Photo-Assisted Corona Poled YLD124/DR1-co-PMMA Electro-Optic Device Using Photoisomerization,” IEEE Photonics Technology Letters, 23(13), 845-847, DOI: 10.1109/LPT.2011.2134854 (2011).

35. Seongku Kim, Wei Liu, Q.Pei, L.R.Dalton, and H. R. Fetterman, "Nonlinear Intermodulation Distortion Suppression in Coherent Analog Fiber Optic Link Using Electro-Optic Polymeric Dual Parallel Mach-Zehnder Modulator,” Optics Express, 19(8), 7865-7871, DOI: 10.1364/QE.19.007865 (2011).

36. Ali Ayazi, Tom Baehr-Jones, Ran Ding, Michael Gould, Woo-Joong Kim, Alexander Spott, Su Huang, Jinghong Luo, Bryan Boyko, Richard Bojko, Alex K.-Y. Jen, and Larry R. Dalton, “Toward a Low-Loss, Ultra-Low Drive Voltage Silicon-Polymer Hybrid Electro-Optic Modulator,” IEEE International Conference on Group IV Photonics-GPP, pp. 252-254 (2011).

37. Ran Ding, Tom Baehr-Jones,Woo-Joong Kim, A. Spott, Maryse Fournier, J.-M. Fedeli, Su Huang, Jingdong Luo, Alex Jen, Larry R. Dalton, and Michael Hochberg, "Sub-Volt Silicon-Organic Electrooptic Modulator with $500 \mathrm{MHz}$ Bandwidth,” IEEE/OSA Journal of Lightwave Technology, 29(8), 1112-1117, DOI: 10.1109/JLT.2011.212344 (2011).

38. William H. Steier and Larry R. Dalton, Polymer Modulators, In Broadband Optical Modulators: Science, Technology, and Applications; A. Chen and E. Murphy, eds.; Taylor \& Francis: New York, NY; USA, 2011, Chap. 9, pp. 221-254.

39. L. R. Dalton, "Electro-Optics," McGraw-Hill Encyclopedia of Science \& Technonology, McGraw-Hill, New York, 2011.

40. Larry R. Dalton and Stephanie J. Benight, “Theory-Guided Design of Organic Electro-Optic Materials,” Polymers, 3, 1325-1351, DOI: 10.3390/polym3031325 (2011).

41. Wenkel Liang, Xiaosong Li, Larry R. Dalton, Bruce H. Robinson, and Bruce E. Eichinger, "Solvents Level Dipole Moments,” J. Phys. Chem. B, 115(43), 12566-12570 DOI:10.1021/jp2069896 (2011).

42. Larry R. Dalton, "Theory-Guided Nano-Engineering of Organic Electro-optic Materials for Integration with Silicon Photonics, Plasmonics, and Metamaterial Devices,” Proc. CLEO-Science and Innovations, Optical Materials and Devices, CW3O, DOI: org/10.1364/CLEO_SI.2013.CV3O.1, ISBN: 978-1-55752-972-5 (2013).

43. Larry R. Dalton and Stephanie J. Benight, "Theory-Inspired Nano-Engineering of Structure, Lattice Dimensionality, and Viscoelasticity of New Polymer and Dendrimer Materials," Molecular Crystals and Liquid Crystals, 554 (1), 4-11 DOI:10.1080/15421406.2012.632733 (2012), available on line at http://www.tandfonline.com/doi/abs/10.1080/15421406.2012.632733.

44. I. Kosilkin, E. A. Hillenbrand, P. Tongwa, A. Fonari, J. Zazueta, M. S. Fonari, M. Antipin, L. R. Dalton, and T. Timofeeva, "Synthesis, Structure, Thermal and Nonlinear Optical Properties of a Series of Novel D- $\pi$-A Chromophores with Varying Alkoxy Substituents,” Journal of Molecular Structure, 1006, 356-365 (2011).

45. Larry R. Dalton, Stephanie J. Benight, Delwin Elder, and Jinsheng Song, "Integration of New Organic Electro-Optic Materials into Silicon and Silicon Nitride Photonics and into Metamaterial and Plasmonic Device Structures,” Proc. Frontiers in Optics, (2011). http://www.opticsinfobase.org/abstract.cfm?URI=FiO-2011-FWBB1.

46. Larry R. Dalton and Stephanie J. Benight, "Systematic Nano-Engineering of Melt-Processable Organic Electro-Optic Materials," Journal of Nanoelectronics and Optoelectronics (2012).

47. Richard S. Kim, Atila Szep, Antao Chen, Haishan Sun, Shaouyan Shi, Don Abersinghe, and Larry R. Dalton, "Fabrication and Characrterization of Hybrid 1x4 Silicon Slot Optical Modulator Array Built on Silicon Photonics and EO Polymer Photonics Technologies for Optical Phase Array Antenna Applications,” Proc. SPIE, 8259, 82590B-115, DOI: org/10.1117/12.913652 (2012).

48. $\quad$ S. J. Benight, D. B. Knorr, Jr., L. E. Johnson, P. A. Sullivan, D. Lao, J. Sun, L. S. Kocherlakota, A. Elangovan, B. H. Robinson, R. M. Overney, and L. R. Dalton, "Nano-Engineering Lattice Dimensionality for a Soft Matter Organic Functional Material,” Adv. Mater., 24 (24), 3263-3268, DOI: 10.1002/adma.201104949 (2012).

49. Stephanie J. Benight, Bruce H. Robinson and Larry R. Dalton, "Nano-Engineering of Molecular Interactions in Organic Electro-Optic Materials,” In: Molecular Interaction, Aurelia Meghea (Ed.), Chapter 8, pp. 133-298, ISBN: 978-953-51-0079, InTech (2012); available from: http://www.intechopen.com/articles/show/title/nano-engineering-ofmolecular-interactions-in-organic-electro-optic-materials.

50. Stephanie J. Benight and Larry R. Dalton, "Nano-Engineered Lattice Dimensionality of Soft Matter Organic Functional Materials: Structure, Order, Viscoelasticity, and Theory,” Nonlinear Optics and Quantum Optics. Concepts in Modern Optics 45 (1), 3-14 (2012).. 
51. S. R. Hammond, J. Sinness, S. Dubbury, K. A. Firestone, Z. Wawrzak, J. B. Benedit, O. Clot, P. J. Reid, and L. R. Dalton, "Molecular Engineering of Nanoscale Order in Organic Electro-Optic Glasses,” J. Mater. Chem., 22, 67526764, DOI: 10.1039/C2JM14915J (2012).

52. Daniel B. Knorr, Jr., Stephanie J. Benight, Brad Krajina, Cheng Zhang, Larry R. Dalton, and Rene M. Overney, "Nanoscale Phase Analysis of Molecular Cooperativity and Thermal Transitions in Dendritic Nonlinear Optical Glasses,” J. Phys. Chem. B, 116 (46), 13793-13805, DOI: org/10.1021/jp307370y (2012).

53. Larry R. Dalton, “Theory-Guided Nano-Engineering of Organic Electro-Optic Materials for Hybrid Silicon Photonic, Plasmonic, and Metamaterial Devices,” Proc. SPIE, 8622, 86220J-1-10, DOI: 10.1117/12.2000246 (2013).

54. Cheng Zhang, Liangie Zhang, Stephanie J. Benight, Benjamin C. Olbricht, Lewis E. Johnson, Bruce H. Robinson, Robert A. Norwood, and Larry R. Dalton, "Shape Engineering to Promote Head-to-Tail Interactions of Electro-Optic Chromophores,” Proce. SPIE, 8827, 882705-1-8, DOI: 10.1117/12.2025676 (2013).

55. David L. K. Eng, Stephen T. Kozacik, Ilya Kosilkin, John. P. Wilson, Dylan D. Ross, Shouyuan Shi, Larry Dalton, Benjamin C. Olbricht, and Dennis W. Prather, "Simple Fabrication and Processing of an All-Polymer Electro-Optic Modulator,” IEEE J. Selected Topics in Quantum Electronics, 19(6), 3401308, DOI: 10.1109/JSTQE.2013.2271239 (2013).

56. R. Palmer, S. Koeber, W. Heni, D. L. Elder, D. Korn, H. Yu, L. Alloatti, S. Koenig, . R. Schindler, W. Bogaerts, M. Pantouvaki, G. Lepege, P. Verheyen, J. Van Campenhout, P. Absil, R. Baets, L. R. Dalton, W. Freude, J. Leuthold, and C. Koos, "High-Speed Silicon-Organic Hybrid (SOH) Modulator with $1.6 \mathrm{fJ} / \mathrm{bit}$ and $180 \mathrm{pm} / \mathrm{V}$ In-Device Nonlinearity,” Proc. 39 ${ }^{\text {th }}$ ECOC 2013 (IET Conference Publications), 622CP, 510-512, DOI: 10.1049/cp 2013 1443, ISBN: 9781849197595 (2013)—best student paper: http://www.ecoc2013.org/student-paper-prize.html.

57. C. Weimann, S. Wolf, D. Korn, R. Palmer, S. Koeber, R. Schmogrow, P. C. Schindler, L. Alloatti, A. Ludwig, W. Heni, D. Bekele, D. L. Elder, H. Yu, W. Bogaerts, L. R. Dalton, W. Freude, J. Leuthold, and C. Koos, "SiliconOrganic Hybrid (SOH) Frequency Comb Source for Data Transmission at 784 Gbit/s,” Proc. ECOC 2013 (IET Conference Publications), 633CP, 777-779, DOI: 10.1049/cp.2013.1532 (2013).

58. C. Weimann, P. C. Schindler, R. Palmer, S. Wolf, D. Bekele, D. Korn, J. Pfeifle, S. Koeber, R. Schmogrow, L. Alloatti, D. Elder, H. Yu, W. Bogaerts, L. R. Dalton, W. Freude, J. Leuthold, and C. Koos, “Silicon-Organic Hybrid (SOH) Frequency Comb Sources for Terabit/s Data Transimission,” Optics Express, 22(3), 3629-3647, DOI: 10.1364/OE.22.003629 (2014).

59. Delwin L. Elder, Stephanie J. Benight, Jinsheng Song, Bruce H. Robinson, and Larry R. Dalton, “Matrix-Assisted Poling of Monolithic Bridge-Disubstituted Organic NLO Chromophores,” Chem. Mater., 26(2), 872-874, DOI: 10.1021/cm4034935 (2014).

60. Larry R. Dalton, Polymers for Nonlinear Optics, Encyclopedia of Polymeric Nanomaterials, Springer-Verlag, Berlin Heidelberg, 2013, DOI: 10.1007/978-3-642-36199-9_11-1.

61. Mathias Lauermann, Robert Palmer, Sebastian Koeber, Philipp C. Schindler, Dietmar Korn, Thorsten Wahlbrink, Jens Bolten, Michael Waldow, Delwin L. Elder, Larry R. Dalton, Juerg Leuthold, Wolfgand Freude, and Christian Koos, “16QAM Silicon-Organic Hybrid (SOH) Modulator Operating with $0.6 \mathrm{~V}_{\mathrm{pp}}$ and $19 \mathrm{fJ} / \mathrm{bit}$ at $112 \mathrm{Gbit} / \mathrm{s}$," IEEE J. Lightwave Technol., in press (2014).

62. R. Palmer, S. Koeber, D. L. Elder, M. Woessner, W. Heni, D. Korn, M. Lauermann, W. Bogaerts, L. R. Dalton, W. Freude, J. Leuthold, and C. Koos, "High-Speed, Low Drive-Voltage Silicon-Organic Hybrid Modulator Based on a Binary-Chromophore Electro-Optic Material,” IEEE J. Lightwave Technol., 32 (16), 2726-2734, DOI: 10.1109/JLT.2014.2321498, ISSN: 0733-8724 (2014).

63. Larry R. Dalton, "Nonlinear optical materials." Kirk-Othmer Encyclopedia of Chemical Technology (2014) in press.

64. Wenwei Jin, Peter V. Johnston, Delwin L. Elder, Andreas F. Tillack, Benjaumin C. Olbricht, Jinsheng Song, Philip J. Reid, Ruimin $\mathrm{Xu}$, Bruce H. Robinson, and Larry R. Dalton, "Benzocyclobutene Barrier Layer for Suppressing Conductamce in Nonlinear Optical Devices during Electric Field Poling,” Appl. Phys. Lett., 104. 243304-1-3, DOI: org/10.1063/1.4884829 (2014).

65. Lewis E. Johnson, Larry R. Dalton, and Bruce H. Robinson, “Optimizing Calculations of Electronic Excitations and Relative Hyperpolarizabilities of Electrooptic Chromophores,” Accounts of Chemical Research, in press, DOI: 10.1021/ar5000727 (2014).

66. Robert Palmer, Sebastian Koeber, Markus Woessner, Delwin L. Elder, Wolfgang Heni, Dietmar Korn, Mathias Lauermann, Wim Bogaerts, Larry R. Dalton, Wolfgang Freude, Juerg Leuthold, and Christian Koos, "High-Speed Silicon-Organic Hybrid (SOH) Modulators with 230 pm/V Electro-Optic Coefficient Using Advanced Materials," Proc. OFC (Optical Fiber Communication Conference, Optical Society of America), M3G.4, High Speed Transmitters and Receivers, DOI: org/10.1364/OFC.2014.M3G.4, ISBN: 978-1-55752-993-0 (2014).

67. Claudius Weimann, Philipp Schindler, Dagmawi Bakele, Robert Palmer, Dietmar Korn, Joerg Pfeifle, Sebastian Koeber, Rene Schmogrow, Luca Alloatti, Delwin L. Elder, Hui Yu, Wim Bogaerts, Larry R. Dalton, Wolfgang Freude, Juerg Leuthoid, and Christian Koos, "Data Transmission at Terabits/s Data Rates Using Silicon-Organic Hybrid (SOH) Frequency Combs,” Proc. OFC (Optical Fiber Communication Conference, Optical Society of America), Th4l.2, Silicon Sources and New Wavelengths, DOI: org/10.1364/OFC.2014.Th4l.2, ISBN: 978-1-55752993-0 (2014). 
68. Matthias Lauermann, Claudius Weimann, Alexander Knopf, Delwin L. Elder, Wolfgang Heni, Robert Palmer, Dietmar Korn, Philipp C. Schindler, Sebastian Koeber, Luca Alloatti, Hui Yu, Wim Bogaerts, Larry R. Dalton, Christian Rembe, Juerg Leuthold, Wolfgang Freude, and Christina Koos, "Integrated Silicon-Organic Hybrid (SOH) Frequency Shifter,” Proc. OFC (Optical Fiber Communication Conference, Optical Society of America), Tu2A.1, RF Photonic Devices, DOI: org/10.1364/OFC.2014.Tu2A.1, ISBN: 978-1-55752-993-0 (2014).

69. Larry R. Dalton, Matthais Lauermann, and Christian Koos, "Nonlinear Optics: Electro-Optic Applications, Chapter 14,” in Organic Semiconductors, Wiley Interscience, New York, 2014-currently under review.

70. C. Koos, J. Leuthold, W. Freude, L. R. Dalton, S. Koeber, R. Palmer, C. Weimann, D. L. Elder, W. Heni, D. Korn, J. Pheifle, S. Wolf, D. Bekele, M. Woessner, L. Alloattie, P. C. Schindler, and S. Koenig, "Femtojoule Modulation and Frequency Comb Generation in Silicon-Organic Hybrid (SOH) Devices," Proc. 16 $6^{\text {th }}$ International Conference on Transparent Optical Networks (2014).

71. Aleksandar Nesic, Robert Palmer, Sebastian Koeber, Dietmar Korn, Swen Koenig, Delwin L. Elder, Larry R. Dalton, Wolfgang Freude, and Christian Koos, "Demonstration of Difference Frequency Generation in a Silicon Slot Waveguide,” Proc. CLEO 2014, STh1l.2 DOI: org/10.1364/CLEO_SI.2014.STh11.2, ISSN: 978-1-55752-999-2 (OSA CLEO Science and Innovations Conference Paper, San Jose, CA, USA, June 8-13, 2014.

72. M. Lauerman, R. Palmer, S. Koeber, P. C. Schindler, D. Korn, T. Wahlbrink, J. Bolten, M. Waldow, D. L. Elder, L. R. Dalton, J. Leuthold, W. Freude, C. Koos, "16QAM Silicon-Organic Hybrid (SOH) Modulator Operated with 0.6 Vpp and 19 fJ/bit at 112 Gbit/s,” Proc. CLEO 2014, SM2G.6, DOI: org/10.1364/CLEO_SI.2014.SM2G.6 ISSN: 978-1-55752-999-2 (OSA CLEO Science and Innovations, San Jose, CA, USA, June 8-13, 2014).

73. M. Lauermann, P. C. Schindler, S. Wolf, R. Palmer, S. Koeber, D. Korn, L. Alloattie, T. Wahlbrink, J. Bolten, M. Waldow, M. Koenigsmann, M. Kohler, D. Malsom, D. L. Elder, L. R. Dalton, J. Leuthold, W. Freude, and C. Koos, "40 GBd 16QAM Modulation at 160 Gbit/s in a Silicon-Organic Hybrid (SOH) Modulator," Proc. CLEO 2014, SM2G.6, DOI: org/10.1364/CLEO_SI.2014.SM2G.6 ISSN: 978-1-55752-999-2 (OSA CLEO Science and Innovations, San Jose, CA, USA, June 8-13, 2014).

74. P. C. Schindler, D. Korn, R. Palmer, W. Heni, S. Koeber, M. Lauermann, S. Wolf, A. Ludwid, R. Schmogrow, D. L. Elder, L. R. Dalton, W. Bogaerts, H. Yu, W. Freude, J. Leuthold, and C. Koos, "Ultra-Short Silicon-Organic Hybrid (SOH) Modulator for Bi-Directional Polarization-Independent Operation,” Proc. CLEO 2014, accepted.

75. Andreas Frank Tillack, Lewis E. Johnson, Meghana Rawal, Larry R. Dalton, and Bruce H. Robinson, "Modeling Chromophore Order: A Guide for Improving EO Performance,” Proc. Mater. Res. Soc., (2014).

76. Meghana Rawal, Kerry Garrett, Andreas Tillack, David P. Shelton, Werner Kaminsky, Bruce E. Eichinger, Bruce H. Robinson, and Larry R. Dalton, "Cross-Conjugation as a Design Motif for Non-Linear Optical Molecules,” Proc. Mater. Res. Soc. (2014).

77. S. Koeber, R. Palmer, M. Lauermann, W. Heni, D. L. Elder, D. Korn, M. Woessner, L. Alloatti, S. Koenig, P. C. Schindler, H. Yu, W. Bogaerts, L. R. Dalton, W. Freude, J. Leuthold, and C. Koos, "Femtojoule Electro-Optic Modulation Using a Silicon-Organic Hybrid Devices,” Light: Science and Applications, in press (2014).

78. Philip A. Sullivan and Larry R. Dalton, "Materials Genome: Organic Electro-Optic Materials,” in Plenary Lectures on Nanoscience and Engineering, James G. Grote and David Andrews, eds. (SPIE Press, Bellingham, WA, 2014).

79. M. Lauermann, R. Palmer, S. Koeber, P. C. Schindler, T. Wahlbrink, J. Bolten, M. Waldow, D. L. Elder, L. R. Dalton, J. Leuthold, W. Freude, and C. Koos, "Low-Power Silicon-Organic Hybrid (SOH) Modulators for Advanced Modulation Formats,” Optics Express, submitted (2014).

80. C. Haffner, W. Heni, Y. Fedoryshyn, J. Niegemann, D. L. Elder, A. Emboras, D. Hillerkuss, B. Baeuerle, A. Josten, F.Ducry, A. Melikyan, M. Kohl, L. R. Dalton, C. Hafner, and J. Leuthold, "High-Speed Plasmonic Mach-Zehnder Modulation in a Waveguide,” Proc. ECOC 2014.

81. P. C. Schindler, M. Lauermann, S. Wolf, D. Korn, R. Palmer, S. Koeber, W. Heni, A. Ludwig, R. Schmogrow, D. L. Elder, L. R. Dalton, W. Bogaerts, H. Yu, W. Freude, J. Leuthold, and C. Koos, "Ultra-Short Silicon-Organic Hybrid (SOH) Moduator for Bidirectional Polarization-Independent Operation,” Proc. ECOC 2014.

82. J. Leuthold, A. Melikyan, L. Alloatti, D. Korn, R. Palmer, D. Hillerkuss, M. Lauermann, P. C. Schindler, B. Chen, R. Dinu, D. L. Elder, L. R. Dalton, C. Koss, M. Kohl, W. Freude, and C. Hafner, "Smaller, Faster and More Energy Efficient Modulators - From Silicon Organic Hybrid to Plasmonic Modulation,” Proc. ECOC 2014.

83. M. Lauermann, P. C. Schindler, S. Wold, R. Palmer, S. Koeber, D. Korn, L. Alloatti, T. Wahlbrink, J. Bolten, M. Waldow, M. Koenigsmann, M. Kohler, D. Malsam, D. L. Elder, P. V. Johnston, N. Phillips-Sylvain, P. A. Sullivan, L. R. Dalton, J. Leuthold, W. Freude, and C. Koss, "High-Speed Silicon-Organic Hybrid Modulators for Advanced Modulation Formats,” IEEE J. Lightwave Technol., in press (2014).

84. Larry R. Dalton, M. Jazbinsek, O. P. Kwon, Peter Gunter, and Philip A. Sullivan, Organic Non-linear Optics: Molecules, Polymers and Crystals, Cambridge University Press, Cambridge, 2012, in final editing. 


\section{Personnel Supported:}

Number of $\mathrm{PI}(\mathrm{s})$ and Co-PI(s) Involved: 2

Number of Post Docs Supported: $\quad 3$

Number of Graduate Students Support: 8

Other Researchers Supported: $\quad 2$

Postdoctoral Fellows/Postdoctoral Professional Staff Supported (Partial Support)

Delwin Elder

Bruce Eichinger

Jinsheng Song

Graduate Students Supported (Partial Support)

Stephanie J. Benight

Lewis Johnson

Kerry Garrett

Andreas Tillack

Ilya Kosilkin

Peter Johnston

Meghana Rawal

Nathan Phillips-Sylvain

Other Researchers Support (Undergraduate Students):

Jeffrey Hurst

George M. Kempel

\section{Publications:}


Number of Publications in Refereed Journals: 76

Number of Publications that Acknowledge AFOSR Support: 48

\section{Consultative/Advisory Functions:}

ADVISORY SERVICE-NON FEDERAL

-Member, Editorial Board, Materials Today

-Chairman/Member, External Advisory Committee, Center for Research and Education on Advanced Materials and Center for Materials Research, Norfolk State University, (2002-)

-Chairman/Member, External Advisory Committee, NSF PREM Center, Norfolk State University

-Chairman/Member, External Advisory Committee, NSF CREST Center, Norfolk State University

-Chairman/Member, External Advisory Committee, NSF RISE Center, Norfolk State University

-Member, External Advisory Committee, NSF IGERT Grant, Norfolk State University

-Member, External Advisory Committee, Norfolk State University MS\&E Ph.D. Progrgam

-Member, External Advisory Committee, Georgia Institute of Technology Department of Chemistry and Biochemistry

-Member, External Advisory Committee, NSF RISE Center, Alabama A\&M University

-Member, Scientific American 50 Awards Advisory Committee

-Member, Board of Visitors, Chemistry Department, University of Alabama

-Guest Editor, Special Issue of the Journal of Physical Chemistry

-Guest Editor, Special Issue of the IEEE Journal of Selected Topics in Quantum Electronics

-Academic Technology Advisory Committee, University of Washington

-Member, Advisotry Committee, ACS Journal Chemistry of Materials

-Advisor, SPIE Newsroom

-Editorial Advisory Committee, Annual Reviews of Physical Chemistry

\section{FEDERAL AND STATE ADVISORY SERVICE}

-Member, Organizing Committee and PI of NSF Grant Supporting the American Chemical Society Symposium on Advancing the Chemical Sciences Through Diversity in Participation, San Francisco 
-Member, NSF CCI Review Panel

-Member, DoE Los Alamos/Sandia Nanotechnology Center Review Panel

-Chairman, Committee of Visitors, EPSCoR Program, OIA, National Science Foundation

-Board Member, NSF/DoE/NIH Open Chemistry Collaborative in Diversity Equity (OXIDE-http://www.oxide.gatech.edu/ver1.0/index.html) (2010-); Presented Invited Keynote Lectures at 2012 and 2013 meetings.

-Member, DoE EFRC Mid-Term Review Panel

-Advisor, Germany's Excellence Initiative and Invited Attendee, DFG Networking Event, New York, New York

-Defense Science Board (DSB)-Advisory Group on Electron Devices (AGED)/Special Government Employee

-Member, Nanotechnology Technical Advisory Group (nTAG) of the President's Council of Advisors on Science and Technology (PCAST); Invited Presenter at U.S.-Korea Forum on Nanotechnology

-Member, National Science Foundation (NSF) Advisory Committee for the Government Performance and Results Act (ACGPA)

-Member, Advisory Committee, Mathematical and Physical Science Directorate, National Science Foundation (MPSAC)

-Member, NSF EPSCOR Panel

-Member, NSF/DoE/NIH Diversity Equity Workshop Planning Committee and invited workshop speaker

-Member, Committee of Visitors, Division of Materials Research, National Science Foundation -Member, Department of Energy INCITE Chemistry Panel; Reviewer for Condensed Matter Physics Panel

-Member, NSF Partnerships for International Research and Education (PIRE) Program NANO Panel

-Member, Committee of Visitors, Chemistry Division, National Science Foundation

-Member, NSF MPSAC Subcommittee on the American Competitiveness Initiative

-Member, NSF MPSAC Subcommittee on Major Facilities

-Member, Chemistry Review Panel, Deutsche Forschungsgemeinschaft (DFG)

-Member, Organizing Committee for the NSF Workshop on Excellence Empowered by a Diverse Academic Workforce: Achieving Racial and Ethnic Equity in Chemistry 
-Invited Participant, Workshop: Building Electronic Function into Molecular Architectures, Arlington, VA

-National Science Foundation Materials Education Workshop, Arlington, VA

-Member, Engineering and Physical Sciences Research Council (EPSRC), England

-Consultant, Arizona Biomedical Research Commission (formerly, Arizona Disease Control Research Commission); Chair, Review Panel for Biomedical Engineering, Imaging, and Sensing

-Member, National Science Foundation Panel on the Technological Challenges of Organic Electronic and Photonic Materials

-Member, National Science Foundation Panel on Sensors and Sensor Systems

-Member, National Science Foundation Workshop on Chemical Bonding Centers

-Member, National Science Foundation "Grand Challenges Workshops" for the National Nanotechnology Initiative

-Member (Ad Hoc), Air Force Scientific Advisory Board

-Member, Pacific Northwest National Laboratory Peer Review Panel for the Energy Science and Technology Directorate

-Oversight Reviewer of the National Research Council Report on Implications of Emerging Micro- and Nanotechnologies

-Member, Pacific Northwest National Laboratory Peer Review Panel for the Nanotechnology Initiative

-Member, National Science Foundation NIRT Review Panel

-Member, National Science Foundation Panel on Information Technology Research (ITR)

-Member, National Science Foundation SBIR/STTR Peer Review Panel

-Member, National Science Foundation IGERT Review Panel

-Member, National Science Foundation Nanotechnology Review Panel

-Reviewer, Arizona Biomedical Research Commission (formerly, Arizona Disease Control Research Commission) for Annual Review of Proposals

Participation/Presentations: (2009-2014)

Conference — 2009-Ohio Innovation Summit, Dayton, OH—Invited Keynote Lecture: "Is Technology Innovation Necessary for Economic Recovery and Long-Term Economic Vitality?” 
Conference-2009-IEEE/LEOS International Conference on Optical MEMS and NanophotonicsInvited Lecture: "Multiple slot waveguides for enhanced biochemical sensing"

Conference-2010-American Chemical Society, August 23, 2010, Invited Presentation: "Engineering Order in Soft Matter Electroactive Materials”

Conference-2010-SPIE-Int. Soc. Opt. Eng.--Invited Lecture: “Active plasmonic and metamaterials and devices"

Conference-2011-Conference on Lasers and Electro-Optics Europe and $23^{\text {rd }}$ European Quantum Electronics Conference, Munich, Germany--Invited Lecture: "Horizontally slotted electro-optic waveguides featuring an organic crystalline slot material”

Conference-2011-CLEO (Conference on Lasers and Electro-Optics, Baltimore, MD)--Invited Lecture: “Au and Ag nano-particle embedded plasmonic metal-slotted polymer electro-optic waveguide modulator”

Conference-2011-SPIE-Int. Soc. Opt. Eng.--Invited Lecture: "Optimization of organic NLO materials for integration with silicon photonic, plasmonic (metal optics) and metamaterial devices”

Conference-2011-8 ${ }^{\text {th }}$ IEEE International Conference on Group IV Photonics-GPP 2011, London, United Kingdom-Invited Lecture: "Toward a low-loss, ultra-low drive voltage silicon-polymer hybrid electrooptic modulator”

Conference - 2011-Frontiers in Optics, OSA - Invited Lecture: "Integration of new organic electro-optic materials into silicon and silicon nitride photonics and into metamaterial and plasmonic device structures"

Conference-2011-11 ${ }^{\text {th }}$ ICFPAM, Pretoria, South Africa--Invited Lecture: “Theory-inspired nanoengineering of structure, lattice dimensionality, and viscoelasticity of new polymer and dendrimer materials”

Conference-2011-ICONO12/ICOPE2011, Trinity College, Dublin, Ireland--Invited Lecture: "Systematic nano-engineering of melt-processable organic electro-optic materials"

Seminar-2011-University of Arizona, November 14, 2011-Invited Presentation: “Theory-guided chromophore (molecular) modification for control of material order and viscoelastic properties or how do we achieve $>300 \mathrm{pm} / \mathrm{V}$ in devices?”

Seminar-2011-Hewlett-Packard, Palo Alto, CA — Invited Presentation: “Optimization of optoelectronics by systematic control of intermolecular interatctions”

Conference-2012-TCCP TP6 Workshop on Organic Metamaterials.--Invited Lecture: “Organic nonlinear optical materials for active metamaterial, plasmonic, silicon nitride and silicon photonic devices”

Workshop-2012-Workshop on Polymers for Optics and Electronics, Case Western Reserve University-Invited Lecture: "A template for the nano-engineering of macromolecular materials with controlled order and dynamics” 
Conference-2012-9 ${ }^{\text {th }}$ SPSJ International Polymer Conference (IPC2012), Kobe, Japan--Invited Lecture: "Theory-guided nano-engineering of order and molecular cooperativity in macromolecular materials"

Distinguished Lecture - 2012 - Department of Energy CNMS Discovery Lecture: "Systematic theoryguided nano-engineering of molecular order, lattice dimensionality, and viscoelastic properties of organic electo-active materials"

University of Tennessee College of Engineering Distinguished Lecture-2012—Knoxville, TN: "Theory-guided systematic nano-engineering of photonic materials and devices"

Conference - 2013-CLEO:2013, San Jose, CA—Invited Lecture: "Theory-guided nano-engineering of organic electro-optic materials for integration with silicon photonics, plasmonics, and metamaterial devices”

Conference-2013-SPIE-Int. Soc. Opt. Eng.--Invited Lecture: "Fabrication and characterization of a hybrid $1 \times 4$ silicon-slot optical modulator incorporating EO polymers for optical phased-array antenna applications"

Conference-2013-SPIE-Int. Soc. Opt. Eng.--Invited Lecture: “Theory-guided nano-engineering of organic electro-optic materials for hybrid silicon photonic, plasmonic, and metamaterial devices"

Conference - 2013-European Conference on Optical Communications, London, United KingdomInvited Lecture: "High-speed silicon-organic hybrid (SOH) modulator with $1.6 \mathrm{fJ} / \mathrm{bit}$ and $180 \mathrm{pm} / \mathrm{V}$ indevice nonlinearity"

Conference - 2013-European Conference on Optical Communications, London, United KingdomInvited Lecture: "Silicon-organic hybrid (SOH) frequency comb source for data transmission at 784 Gbit/s”

Conference-2014-Optical Fiber Communications Conference, OSA, 2014—Invited Lecture: "Highspeed silicon-organic hybrid (SOH) modulators with $230 \mathrm{pm} / \mathrm{V}$ electro-optic coefficient using advanced materials"

Conference - 2014-Optical Fiber Communications Conference, OSA, 2014—Invited Lecture: "Data transmission at terabit/s data rates using silicon-organic hybrid (SOH) frequency combs"

Conference-2014-Optical Fiber Communications Conference, OSA, 2014—Invited Lecture: "Integrated silicon organic hybrid (SOH) frequency shifter"

Conference - 2014- $16^{\text {th }}$ International Conference on Transparent Optical Networks 2014-Invited Lecture: "Femtojoule modulation and frequency comb generation in silicon-organic hybrid (SOH) devices"

Conference - 2014-CLEO:2014, San Jose, CA—Invited Lecture: "Demonstration of difference frequency generation in a silicon slot waveguide"

Conference - 2014-CLEO:2014, San Jose, CA—Invited Lecture: “16QAM silicon-organic hybrid (SOH) modulator operated with $0.6 \mathrm{Vpp}$ with $19 \mathrm{fJ} / \mathrm{bit}$ at $112 \mathrm{Gbit} / \mathrm{s}$ " 
Conference-2014-CLEO:2014, San Jose, CA—Invited Lecture: “40 GBd 16QAM modulation at 160 Gbit/s in a silicon-organic hybrid (SOH) modulator”

Conference-2014-CLEO:2014, San Jose, CA-Invited Lecture: "Ultra-short silicon-organic hybrid (SOH) modulator for bi-directional polarization-independent operation”

Conference-2014-40 ${ }^{\text {th }}$ European Conference on Optical Communications, Cannes, France-Invited Lecture: "High-speed plasmonic Mach-Zehnder modulation in a waveguide"

Conference-2014-40 ${ }^{\text {th }}$ European Conference on Optical Communications, Cannes, France-Invited Lecture: "Ultra-short silicon-organic hybrid (SOH) modulator for bidirectional polarization independent operation”

Conference-2014-40 ${ }^{\text {th }}$ European Conference on Optical Communications, Cannes, France-Invited Lecture: "Smaller, faster and more energy efficient modulators-From silicon organic hybrid to plasmonic modulation”

\section{Interactions/Transitions:}

Transition 1

a. Dalton, UW, b. Data for organic electro-optic materials, c. William Krug/Boeing Phantom Works., 253657-8018 d. For a development of a research program for handling both digital and analog data exploiting WDM techniques and explicitly wavelength selective filter and beam steering concepts. $1 \times 4 \times 1$ ROADM device delivered to NAVAIR-results published in IEEE Journal of Lightwave Technology.

Transition 2

a. Dalton, UW, b. Data relating to polymeric electro-optic materials and processing protocols, c. Susan Ermer/Lockheed Martin Corporation, 650-424-3131 d. For prototyping high frequency, low drive voltage modulators.

Transition 3

a. Dalton, UW, b. Polymeric electro-optic materials and processing protocols, c. Lumera Corporation (Gigoptix), 425-415-6616 d. For electro-optic device fabrication and consideration for establishing an electro-optic materials production facility.

Transition 4

a. Dalton, UW, b. Information related to organic electro-optic materials, c. Ray Chen/UT-Austin Microelectronics Center and Radiant Photonics, 512-338-4942 d. For electro-optic device fabrication and evaluation.

Transition 5 
a. Dalton, UW, b. Polymeric electro-optic materials and processing protocols, c. James G.

Grote/AFRL/MLPO, 937-255-4474 ext $3263 \mathrm{~d}$. For use with conducting cladding materials developed by AFRL.

\section{Transition 6}

a. Dalton, UW, b. Polymeric electro-optic materials and processing protocols, c. TACAN Corporation, 760-438-1010 ext 3278 d. Polymeric electro-optic materials for device fabrication.

Transition 7

a. Dalton, UW, b. Polymeric electro-optic materials and processing protocols, c. Professor Robert Norwood, University of Arizona, 520-626-0936 d. Polymeric electro-optic materials for evaluation

Transition 8

a. Dalton, UW, b. Polymeric electro-optic materials and processing protocols, c. Professor Dennis Prather, 302-831-8170, d. Polymeric electro-optic materials for sensor and RF photonic applications

\section{New discoveries, inventions, or patent disclosures:}

Significant improvements have been made in organic electro-optic and sensor materials and a new paradigm for developing materials with further improvement in properties has been validated. Integration

of organic optoelectronic materials with silicon photonics, plasmonic, and metamaterial deice architectures has been demonstrated.

Honors/Awards: (Larry Dalton, Lifetime)

\section{Professional Society Awards}

•2011 Linus Pauling Award and Medal (American Chemical Society)

-2008 Lifetime Achievement Award, SPIE-International Society of Optics and Photonics

•2006 IEEE/LEOS William Streifer Scientific Achievement Award

-2003 Chemistry of Materials Award of the American Chemical Society

•1996 Richard C. Tolman Medal (American Chemical Society)

-QEM (Quality Education for Minorities)/MSE (Mathematics, Science, and Engineering) Network 2005 Giants in Science Award

\section{Election to Fellow Status of Professional Societies}

-Fellow, Optical Society of America

-Fellow, SPIE-International Society of Optics and Photonics

-Fellow, American Association for the Advancement of Science 
-Fellow, American Chemical Society (Inaugural Class)

-Fellow, Materials Research Society

-Senior Member, IEEE

-Awarded Lifetime Membership SPIE (sponsored by SPIE and AFRL)

-Elected to the Washington State Academy of Sciences (2012)

\section{Endowed Lectureships}

-University of Tennessee-Knoxville College of Engineering Distinguished Lecturer (March 2012) -2012 Department of Energy CNMS Discovery Lecturer, Oak Ridge National Laboratory -2011 Lloyd N. Ferguson Distinguished Lecturer, California State University, Los Angeles - University of Utah, College of Science, Frontiers of Science Lectures, Davern/Gardner Laureateship (2007)

-Dow/Karabatsos Lecture Series and the Alumni Distinguished Lectureship, Michigan State University (2005)

-AFRL Materials, Manufacturing \& Enabling Technologies Wright Centennial Lecture, Dayton, 2004

•2003 Eastman Lecturer, University of Akron

-2002 Inaugural Lecturer of the National Science Foundation Distinguished Lecture Series

-Paul C. Cross Lectureship, University of Washington, Seattle, WA (1996)

-NASA Lecturer, Fifty-Fourth Frontiers in Chemistry Lecture Series (1995), Case Western Reserve University

\section{Othe Awards including University, Federal and Private Foundation Awards}

-Larry Dalton Festschrift Issue of the Journal of Physical Chemistry (Vol. 112, no. 21, May 29, 2008), American Chemical Society http://pubs.acs.org/toc/jpccck/112/21

-Award honoring the contributions of Larry R. Dalton to Research and Education sponsored by Norfolk State University, the Center for Materials Research, and the Materials Research Society Student Chapter (July 5, 2012)

-Special Symposium on Nonlinear Optical Polymers (SPIE Optics + Phontonics Conference, San Diego 2008) honoring the contributions of Professor Larry Dalton

-2000 Michigan State University Distinguished Alumni Award 
-The 1990 University of Southern California Associates Award for Creativity in Research and Scholarship

•1986 Burlington Northern Foundation Faculty Achievement Award

-SCEEE Fellow (81)

-NIH Research Career Development Awards (Two Awards, one at Stony Brook, one at Vanderbilt, 76-81)

-Camille and Henry Dreyfus Teacher-Scholar Award (75-77)

-Alfred P. Sloan Fellowship (74-77)

\section{Honors and Awards: Co-Pl:}

Bruce H. Robinson (Awards 2009-2014)

- Elected, Fellow of the American Association for the Advancement of Science

\section{Awards Received by Graduate Students Working on This Project:}

-Stephanie Benight, SPIE-International Society for Optics and Photonics Educational Scholarship; Invited Participant at the 2009 Meeting of Nobel Laureates in Landau, Germany 